\title{
Influence of Gold Nano-bipyramid Dimensions on Strong Coupling with Excitons of Monolayer MoS2
}

\author{
Julia Lawless ${ }^{1}$, Calin Hrelescu ${ }^{1}$, Carolyn Elliott ${ }^{1,2}$, Lisanne Peters ${ }^{3}$, Niall McEvoy ${ }^{3}$ and A. Louise \\ Bradley ${ }^{1,2 *}$
}

1. School of Physics and AMBER, Trinity College Dublin, College Green, Dublin 2, Ireland

2. IPIC, Tyndall National Institute, Cork, Ireland

3. School of Chemistry and AMBER, Trinity College Dublin, College Green, Dublin 2, Ireland

\section{KEYWORDS: Rabi Splitting, Plasmon, Exciton, Polariton, Bipyramid, $\mathrm{MoS}_{2}$}

ABSTRACT: Rabi splitting between the longitudinal plasmon of a gold nano-bipyramid and the A exciton of monolayer $\mathrm{MoS}_{2}$ is observed at room temperature. The dependence of the Rabi splitting on the physical dimensions of the nano-bipyramid is reported. The impact of bipyramid length, aspect ratio and tip radius on the coupling strength are investigated. The mode volume of the nanoresonator is significantly reduced due to the sharp tips of the bipyramid, and the Rabi splitting increases with tip sharpness. The results also reveal that greater Rabi splitting is observed for larger bipyramids, contrasting with results perviously reported for different nanoresonator shapes. This shows, for the first time, how the magnitude of the splitting has a different response for particular nanoresonators when tuning the size, without increasing the number of excitons coupled into the system. The Rabi splitting, at zero energy detuning between plasmon and A exciton, increases from $\sim 55$ $\mathrm{meV}$ with a $70 \mathrm{~nm}$ long bipyramid to $\sim 80 \mathrm{meV}$ with a $100 \mathrm{~nm}$ long bipyramid. The

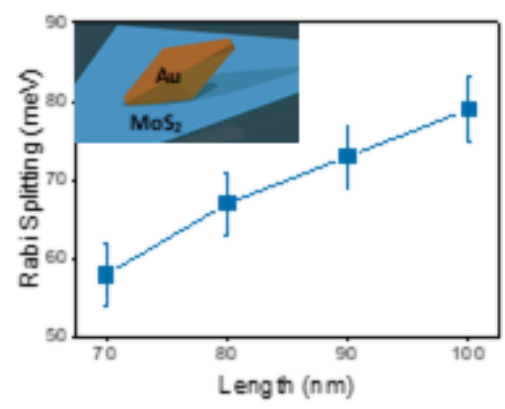
increase in coupling strength with size arises due to increasing confinement of the field enhancement at the bipyramid tip.

\section{Introduction}

In the strong coupling regime, the rate of coherent energy exchange between a quantum emitter and an optical resonator mode is quicker than their intrinsic dissipation rates. In contrast to the weak coupling regime, known as the Purcell effect ${ }^{1}$, the energy of the strongly coupled system is not only enhanced or quenched but splits into two entirely new eigenstates (polaritons). This so-called Rabi splitting has a large range of applications. ${ }^{2}$ These include the control of chemical reactions and modification of the chemical reaction rates, ${ }^{3-5}$ applications in low threshold lasers, ${ }^{6}$ optical switching ${ }^{7}$ and quantum information processing. ${ }^{8}$ An in-depth understanding of the conditions necessary to achieve strong coupling with nanostructures is crucial to pave the road towards technological applications of plasmon-exciton strong coupling.

Previously, the observation of strong coupling was limited to experiments at cryogenic temperatures. ${ }^{9-13}$ With the development of nanoscience, various photonic and plasmonic nanostructures were used to achieve strong coupling at room temperature without the need for a closed optical cavity. ${ }^{13-18}$ For instance, strong coupling was observed in nanosystems consisting of a large quantity of randomly oriented J aggregates interacting with the modes of plasmonic nanostructures. ${ }^{19-21}$

As an alternative to J aggregates, transition metal dichalcogenides (TMDCs) have shown themselves to be an excellent platform for achieving Rabi splitting. ${ }^{14,16,22-28}$ In the bulk, they are a stack of two-dimensional layers, held together by van der
Waals forces. ${ }^{29,30}$ In the single-layer limit, TMDCs exhibit different optical properties, with many transitioning from an indirect to a direct band-gap. ${ }^{31,32}$ In particular, monolayers of TMDCs have tightly bound excitons with high oscillator strengths, due to high quantum confinement. This arises as the thickness of the monolayer is smaller than the Bohr radius. ${ }^{33-36}$ TMDC monolayers are of particular interest for optoelectronic applications since their optical properties are relatively stable and can be tuned with various methods, including electrically. ${ }^{16,37}$ Strong coupling has been achieved with many systems involving TMDCs as the quantum emitter, ${ }^{38}$ including, for example a layer of a TMDC in a microcavity with the cavity thickness used to tune the coupling. ${ }^{23,39,40}$ Lattices of plasmonic nanoparticles have also been used to investigate Rabi splitting, with the particle size and the lattice constant both used to tune the plasmonic resonances. ${ }^{23,41,42}$

A variety of investigations have been carried out to study the strong coupling of individual plasmonic nanoparticles with TMDCs on a substrate, ${ }^{16,43,44}$ allowing for a more detailed examination of the interaction of a few excitons with the confined optical modes. Such studies are of major interest for quantum optics. However, the excitons of TMDC monolayers have a high in-plane dipole transition moment but extremely reduced or non-existent out-of-plane dipole moments. Consequently, in several nanoparticle TMDC systems, such as particle-on-film configurations, nanoparticle dimer, spherical particles and even nanorods on TMDC monolayers, the local electric field and the dipole moment are far from being colinear. This misalignment 

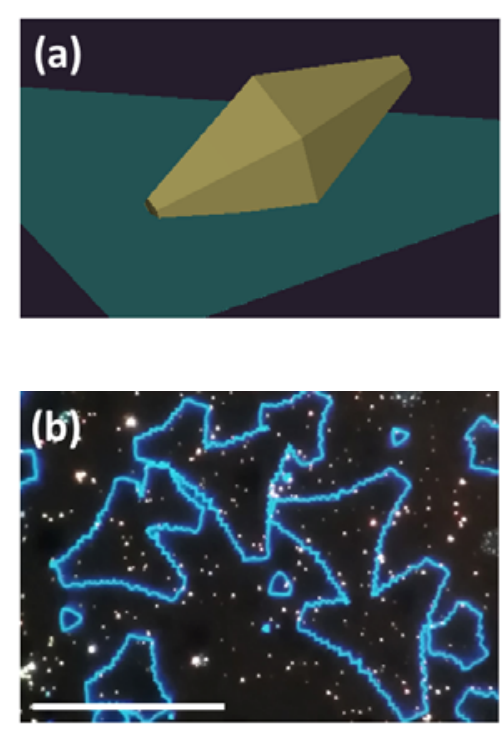
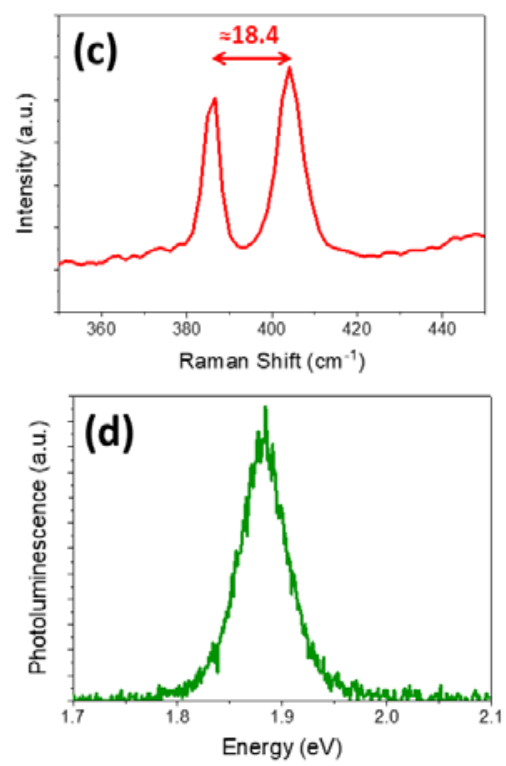
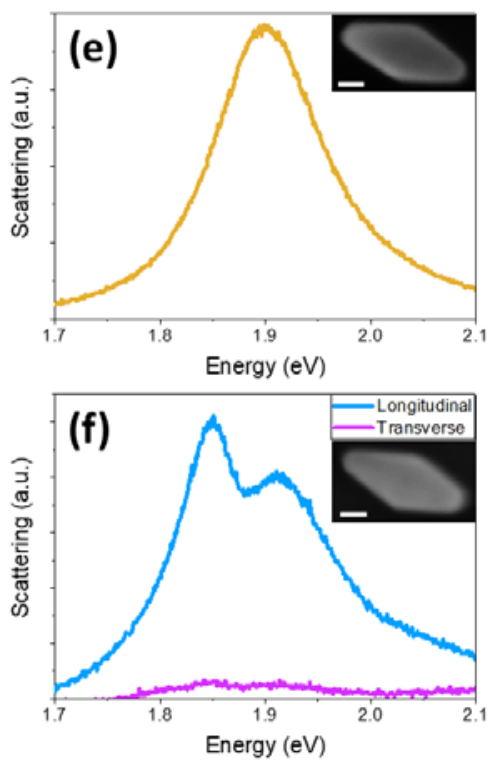

Figure 1. (a) Schematic showing a single Au bipyramid lying on a $\mathrm{MoS}_{2}$ flake on a $\mathrm{SiO}_{2} / \mathrm{Si}$ substrate. (b) Dark field microscopy image of Au bipyramids on $\mathrm{MoS}_{2}$ flakes (white scale bar shows $50 \mu \mathrm{m}$ ). (c) and (d) Typical Raman and PL spectra of MoS flakes used. (e) Scattering spectrum of a single bipyramid directly on a $\mathrm{SiO}_{2} / \mathrm{Si}$ substrate. (f) Polarization resolved scattering spectra of a single Au bipyramid on monolayer MoS2. Insets show corresponding SEM images (white scale bars show $20 \mathrm{~nm}$ ).

can make strong coupling between plasmonic nanoparticles and monolayers impossible. ${ }^{28}$ Additionally, other reports on strong coupling between single metal nanoparticles and quantum emitters have found that the coupling strength decreases with increased metal volume of the nanoparticle ${ }^{16,17}$ but little research has been conducted to find if this remains true for varying particle shapes.

Recently, Stührenberg et al. ${ }^{14}$ reported on an elegant method to overcome the misalignment between the local fields induced by nanoparticles and the in-plane dipole moments of the TMDC monolayers using nanoparticles with bipyramidal shape. In that case the dependence of the Rabi splitting on the aspect ratio of the bipyramids and the number WSe 2 layers was investigated. Rabi splitting greater than $100 \mathrm{meV}$ was reported for Au bipyramids on mono- and multilayer WSe2.

Motivated by the report of Stührenberg et al., ${ }^{14}$ we investigate the interaction of the A excitons of a $\mathrm{MoS}_{2}$ monolayer with gold nano-bipyramids. $\mathrm{MoS}_{2}$ was chosen as the TMDC to couple to the bipyramids in this system due to the lower temperatures required for its synthesis compared with $\mathrm{WSe}_{2},{ }^{45}$ as well as its $\mathrm{A}$ exciton energy being within the visible range. ${ }^{29,46}$ The dependence of the strong coupling between the longitudinal plasmon of the Au bipyramids and the A exciton of the monolayer of $\mathrm{MoS}_{2}$ for varying bipyramid lengths is measured at single nanoparticle level. We present new insights on how the tip radii and overall bipyramid size affect strong coupling, since nanobipyramids on TMDC monolayers are the ideal candidates to enable the investigation of few excitons strongly coupled to the spatially confined optical mode, i.e. the electric field enhancement in the hot spot at the tips of the bipyramid (as suggested by Stührenberg et al. ${ }^{14}$ ). Here we present evidence showing that in the case of bipyramid nanoresonators the coupling strength increases with a higher metal volume, due to the electric field enhancement at the tip of the larger bipyramids. This is in contrast to previous reports where the coupling strength decreases with increased metal volume of the nanoresonator ${ }^{16,17}$
The coupled oscillator model can be used to describe the strong coupling system. In a strongly coupled system, the plasmon and exciton hybridise into two new energetical eigenstates, namely the upper and lower polariton. The energies of the upper and lower polaritons ( $\omega_{u p}$ and $\omega_{l p}$ respectively) are given by the following equation ${ }^{13,47}$

$$
\omega_{\mathrm{up} / \mathrm{lp}}=\frac{\omega_{\mathrm{pl}}+\omega_{\mathrm{ex}}}{2} \pm \sqrt{\mathrm{g}^{2}+\frac{1}{4}\left[\Delta \omega-\frac{\mathrm{i}}{2}(\Delta \gamma)\right]^{2}}
$$

where $\omega_{\text {ex }}$ is the energy of the A exciton, $\omega_{\mathrm{pl}}$ is the energy of the plasmon, $g$ is the coupling strength, $\Delta \omega=\omega_{p l}-\omega_{e x}$ is the energy detuning and $\Delta \gamma=\gamma_{p l}-\gamma_{e x}$, where $\gamma_{p l}$ is the plasmon dissipation rate and $\gamma_{e x}$ is the exciton dissipation rate.

Gold nano-bipyramids offer several advantageous properties to achieve strong coupling. The longitudinal plasmon resonance of Au nano-bipyramids can be easily tuned to overlap with the energy of the A exciton by varying dimensions of the bipyramid. A spectrally sharp plasmon resonance, which is equivalent to a low plasmon dissipation rate $\gamma_{p l}$, is important for strong coupling, since the coupling strength $g$ must exceed both, the plasmon and the exciton dissipation rate ret $^{15-52}$

$$
g>\gamma_{p l}, \gamma_{e x}
$$

Another important effect of the shape of the bipyramids is their tuneable, sharp tips, causing a large localization of the electric field and a substantial field enhancement at the tips. This provides the opportunity to increase the coupling strength. ${ }^{17,19,53}$ The sharp tips also offer the ideal platform for reducing the mode volume of the resonator. On a substrate only one of ten facets of the bipyramid touches the surface (see figure 1a). This further reduces the mode volume of the resonator as only one sharp tip is in contact with the TMDC monolayer, and hence only one hot spot interacts with the quantum emitter. The coupling constant, $\mathrm{g}$, depends on the mode volume as described by the following equations: $:^{9,10,13}$

$$
g=\sqrt{N} \mu_{e}\left|E_{v a c}\right|
$$



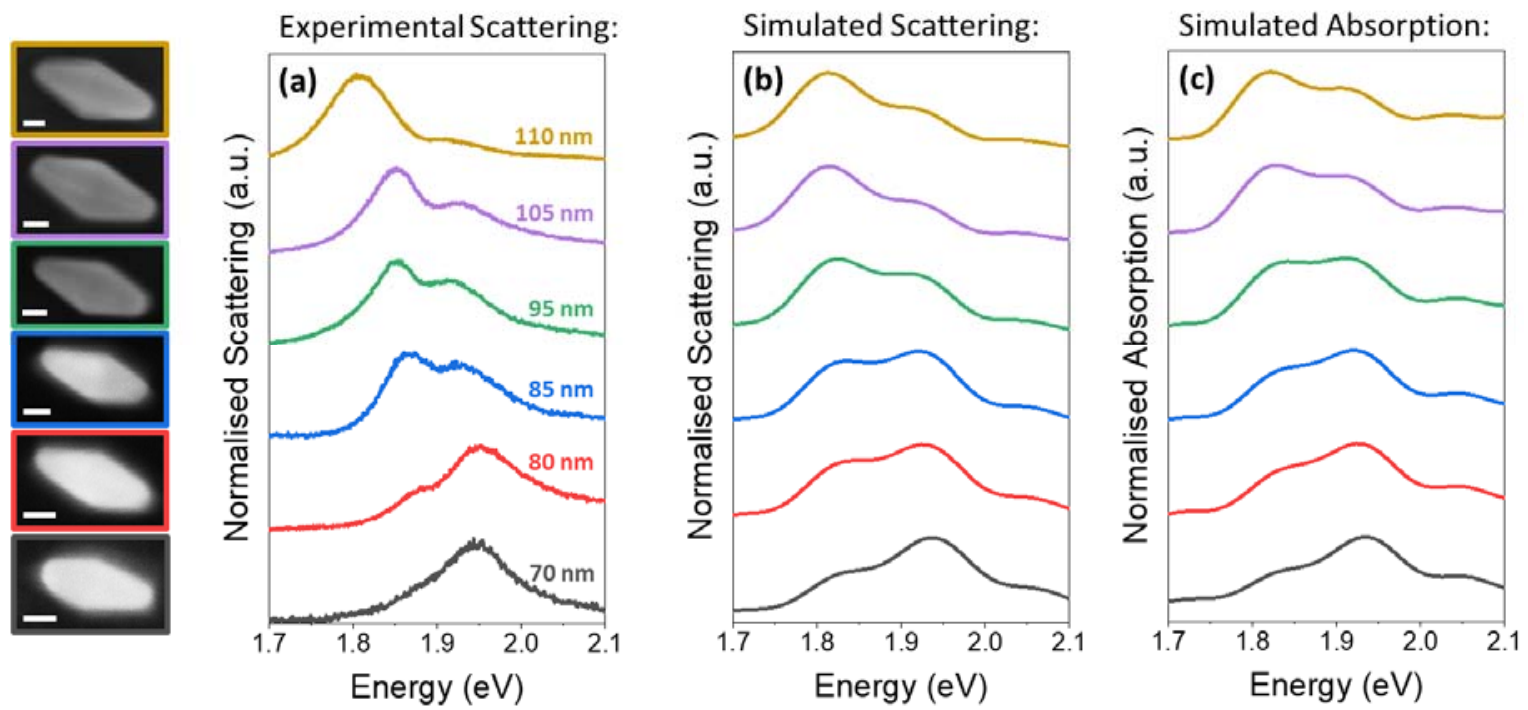

Figure 2. (a) Experimental scattering from $\mathrm{Au}$ bipyramids of varying lengths with aspect ratio $\approx 2.3$ on monolayer MoS2. Corresponding SEM images are shown to the left, with scale bars corresponding to $20 \mathrm{~nm}$. (b) and (c) show the simulated scattering and absorption spectra of the same bipyramids, respectively.

and

$$
E_{v a c} \propto \frac{1}{\sqrt{V}}
$$

where $\mathrm{N}$ is the number of emitters coherently contributing to the interaction in the cavity, $\mu_{e}$ is the transition dipole moment of the quantum emitter, $E_{v a c}$ is the vacuum field and $\mathrm{V}$ is the mode volume of the resonator. This is defined as ${ }^{54}$

$$
\mathbf{V}=\frac{\int \varepsilon \mathbf{E}^{2} \mathbf{d V}}{\operatorname{Max}\left(\varepsilon \mathbf{E}^{2}\right)}
$$

where $\varepsilon$ is the permittivity and $\mathrm{E}$ is the electric field strength.

\section{Results and Discussion}

The $\mathrm{MoS}_{2}$ was grown in monolayer flakes by chemical vapour deposition (CVD) on a $300 \mathrm{~nm} \mathrm{SiO} 2$ layer on a $\mathrm{Si}$ substrate. CVD was chosen as the fabrication method due to its higher crystallinity and larger flake sizes in comparison with other methods. ${ }^{55,56}$ The gold nano-bipyramids were synthesized from $\mathrm{Au}$ penta-twinned seeds ${ }^{57,58}$ using a wet chemistry approach. ${ }^{57}$ Cetyl trimethylammonium bromide (CTAB) was used as the stabilizing ligand. A high degree of control and uniformity of size and plasmon resonance energy was achieved within a batch of nano-bipyramids. Several batches of nano-bipyramids with different overall size, length, tip radius and aspect ratio were synthesized. The Au nano-bipyramids with a low concentration were drop-casted on $\mathrm{MoS}_{2}$ flakes for single particle measurements. This allowed for in-depth investigation of the effect of the bipyramid's morphology on the strong coupling in the nano-bipyramid-MoS $\mathrm{S}_{2}$ system. All scattering spectra were measured under white light excitation at single particle level using a dark field microscope (see figure 1b). Each scattering spectrum was subsequently correlated with a scanning electron microscopy (SEM) image of the same nano-bipyramid, revealing the corresponding bipyramid's dimensions.

Prior to the bipyramid deposition, the $\mathrm{MoS}_{2}$ flakes were characterized via Raman spectroscopy to ensure that they were monolayer (see figure 1c). The characteristic E' and $A^{\prime}{ }_{1}$ Raman peaks were observed to be $18.4 \mathrm{~cm}^{-1}$ apart, confirming monolayer thickness of the flakes. ${ }^{46,59-61}$ Figure $1 \mathrm{~d}$ shows a typical photoluminescence (PL) spectrum of the monolayer flakes, later used in the scattering measurements. The large PL peak corresponds to the energy of the A exciton, matching the absorption of the A exciton in both energy position and width. ${ }^{29}$ It can therefore be attributed to a direct-gap luminescence. ${ }^{29}$ To achieve strong coupling, the plasmons of the Au nano-bipyramids were tuned to energetically overlap with the A exciton, i.e. the PL peak.

A slight blue shift of the PL peak was observed after the deposition of bipyramids onto the flakes, indicating a change in the exciton energy. The A exciton peak shifted from $1.82 \mathrm{eV}$ to $1.88-1.90 \mathrm{eV}$. This effect has been previously reported in a similar system using $\mathrm{WSe}_{2} .{ }^{62}$ As in that case, it was found that the PL peak was slightly blue shifted, but the intensity and linewidth were not significantly affected. This observation excluded the hypothesis that the PL shift was due to trionic or defect-related emission. ${ }^{62}$ In our simulations, a similar shift of the Rabi splitting dip to a new, higher energy was observed with the addition of a layer between the bipyramids and $\mathrm{MoS}_{2}$ mimicking the CTAB-ligand.

Typical scattering spectra measured from a single bipyramid directly on the $\mathrm{SiO}_{2} / \mathrm{Si}$ substrate and on a monolayer of $\mathrm{MoS}_{2}$ are shown in figures 1e and 1f, respectively. Both bipyramids have the same dimensions of $95 \mathrm{~nm} \times 40 \mathrm{~nm}$. Insets show the corresponding SEM images. The longitudinal plasmon of the bipyramid on top of an $\mathrm{MoS}_{2}$ flake is clearly seen to be split into two different eigenstates. The scattering for the polarization corresponding to the transverse mode was also measured. The scattering is much weaker and no peaks in the given energy range are evident in the transverse case. It is clear, therefore, that both observed peaks originate from the Rabi splitting due to the coupling of the longitudinal plasmon mode with the A exciton. The plasmon resonance energy of the Au bipyramids were tuned by adjusting their lengths and widths. A longer bipyramid or one with a higher aspect ratio has a lower energy plasmon resonance 

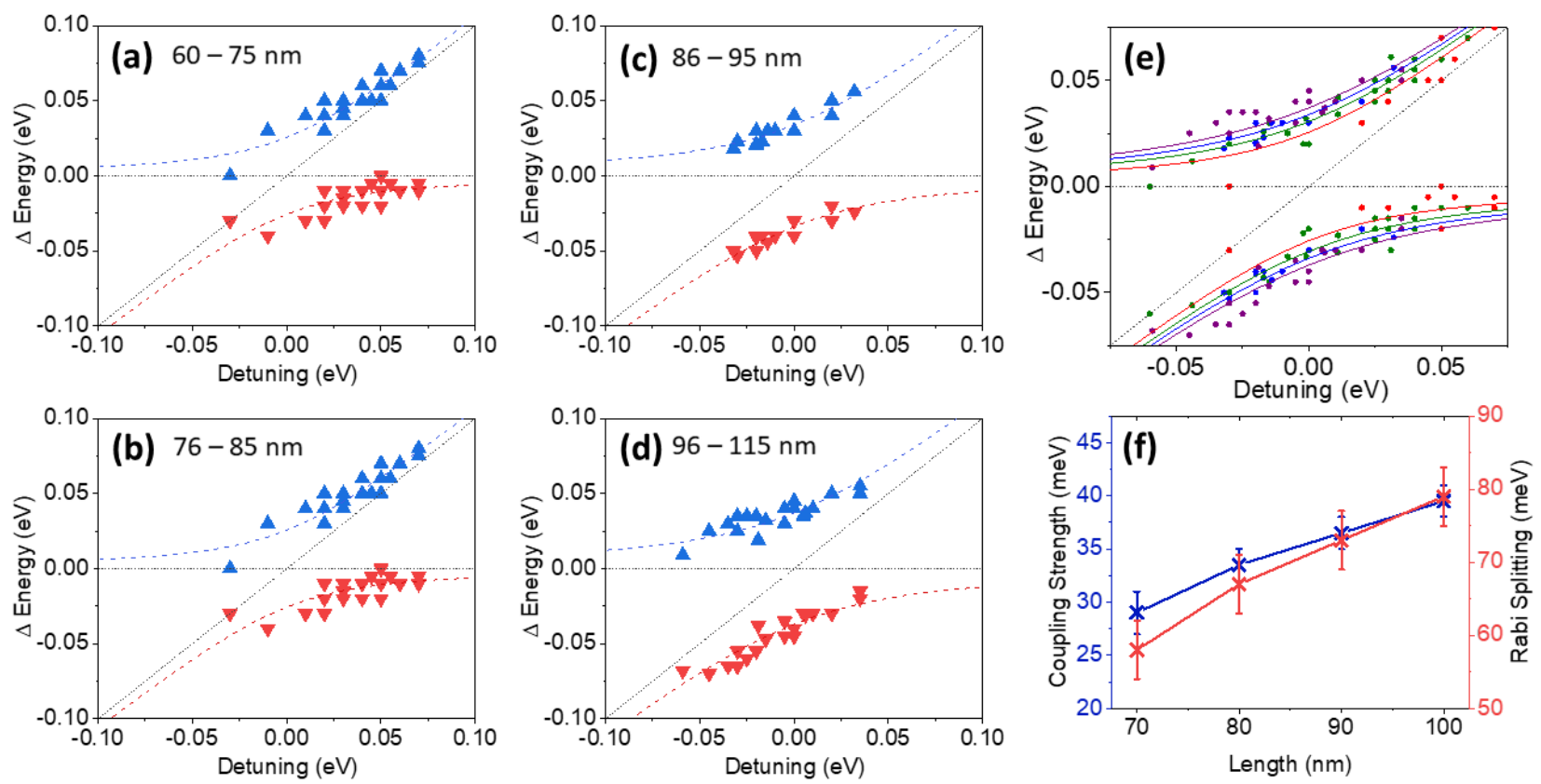

Figure 3. (a)-(d) Experimentally observed Rabi splitting using bipyramids of various sizes coupled to monolayer MoS2. The plots show the difference in energy between the polaritons and the original plasmon plotted against the detuning of the original plasmon from the A exciton in $\mathrm{MoS}_{2}$. The black horizontal and diagonal dashed lines show the energy of the A exciton in MoS and plasmon energy of the bipyramids, respectively. The blue triangles show the energy of the upper polariton and the red triangles show the energy of the lower polariton. The dashed blue and red lines show the fit calculated from the coupled oscillator model (equation 1). (e) Rabi splitting plot of all data, with colours red, green, blue and purple showing the smallest to largest bipyramids, respectively. (f) Plot of coupling strength and Rabi splitting value at zero detuning for each size of bipyramids, estimated from the lines of best fit in plots a-d. The error bars indicate the range of values for coupling strength for which the average difference between the predicted and experimental energies of the polaritons is less than $2 \mathrm{meV}$. The corresponding Rabi splitting is then calculated from the coupling strength.

compared to a shorter bipyramid or one with a lower aspect ratio (see figure $\mathrm{S} 1$ ). The radius tip of the bipyramids was also seen to have an effect, blue shifting the plasmon resonance when it was increased. Sharper tips also produce stronger coupling between the plasmon and exciton in this system.

Figure 2 shows the scattering spectra of six different bipyramids coupled to a monolayer of $\mathrm{MoS}_{2}$. The plasmon mode has been tuned from low to high energy through the A exciton PL energy. The bipyramids each have a similar aspect ratio of $\approx 2.3$ and varying length between 70 and $110 \mathrm{~nm}$. As previously discussed, the plasmon resonance of a bipyramid changes with bipyramid length, a larger bipyramid having a lower energy plasmon resonance. Figure 2a shows the experimental scattering spectrum for each of the bipyramids. The corresponding SEM images are shown to the left. As can be seen in the figure 2a, this has a large effect on the two polaritons. The longest bipyramid has the lowest energy plasmon resonance and gives larger scattering by the lower polariton when coupled to the $\mathrm{MoS}_{2}$. It is seen that the shortest bipyramid has the highest plasmon resonance energy, and more light is scattered in the upper polariton in the coupled system.

Real strong coupling can be confused with Fano-like interferences when looking at scattering spectra. ${ }^{17,63}$ Previous studies have found that only absorption measurements can be used to prove the presence of Rabi splitting. ${ }^{15,17}$ An absence of splitting in the absorption would indicate that the effect seen in the scattering spectra is due to a Fano resonance. ${ }^{15}$ Finite difference time domain (FDTD) simulations were carried out to reproduce the experimental scattering spectra (see figure $2 b$ ). In both the simulated and experimental data, the two polaritons are visible with the dip in the middle occurring at the exciton energy. The simulations were also used to calculate the absorption spectra for each of the bipyramids (see figure $2 \mathrm{c}$ ). This was to ensure that the peaks in the scattering spectra were not appearing due to interference. The absorption peaks were seen to have close resemblance to the scattering peaks, confirming that the observed effect is because of Rabi splitting. ${ }^{15,17,63}$

An extra peak is visible in the simulated spectra at about 2.05 $\mathrm{eV}$. This peak arises due to the presence of the B exciton (see figure $\mathrm{S} 2$ ). The $\mathrm{B}$ exciton is not seen to have as visible an effect in the experimental spectra. The simulated spectra are seen to have slightly broader peaks than in the experimental data. The dielectric function used for the simulations ${ }^{64}$ can differ slightly from the experimental $\mathrm{MoS}_{2}$. Altering the dielectric function to have higher amplitude for the excitonic peaks would narrow the simulated the polariton peaks. Therefore, the slightly broader simulation spectral peaks are attributed to a small discrepancy of the dielectric function, which is a close approximation to the experimental $\mathrm{MoS}_{2}$

Separate anti-crossing plots were made using the experimental data for the different sized bipyramids. The size categories studied were those of bipyramids with length between $60-75 \mathrm{~nm}$, 76-85 nm, 86-95 nm and 96-115 nm, shown in figure 3a-d. Each size category included bipyramids on several different samples, ensuring that any trends found in the plots were due to the different bipyramid sizes. The bipyramids studied had varying aspect ratios, allowing for a range of plasmon energies to be studied for each size. The polariton energies are shown as the difference in energy, $\Delta E n e r g y$, of the scattering peaks from the A exciton in $\mathrm{MoS}_{2}$. This accounts for slight differences $(0.02 \mathrm{eV})$ 

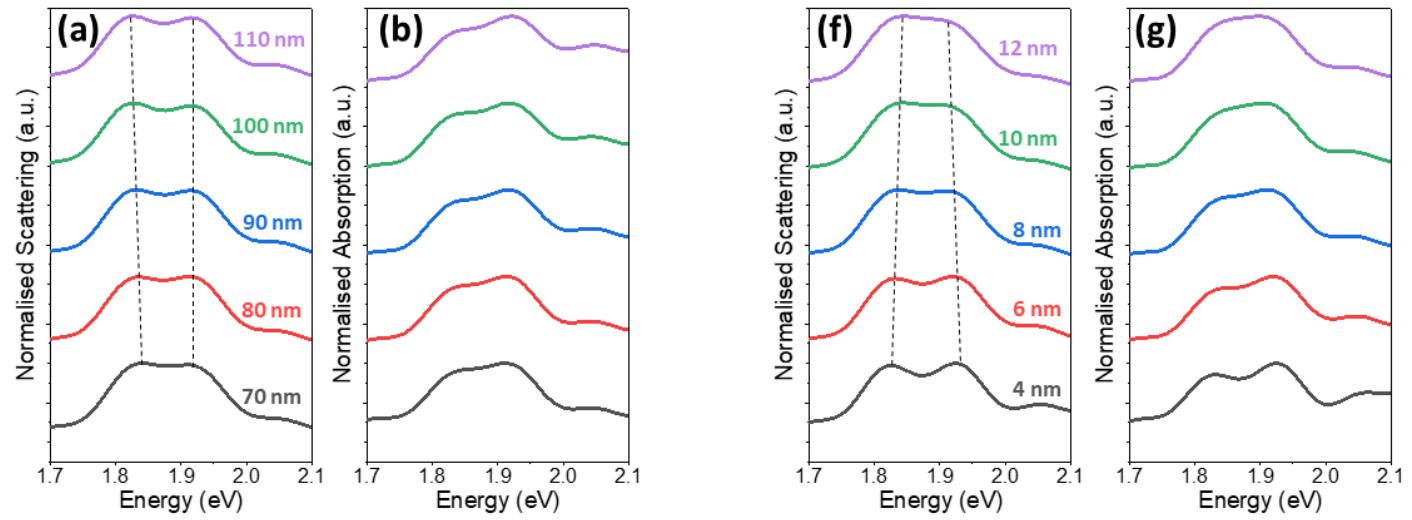

(c)

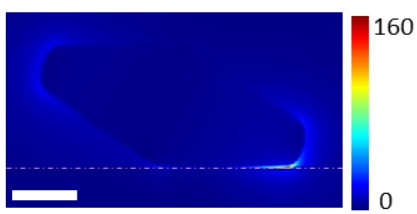

(d)

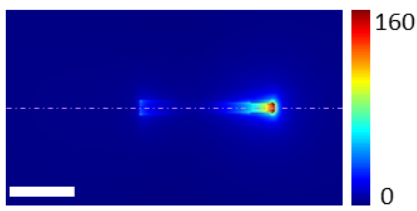

(e)

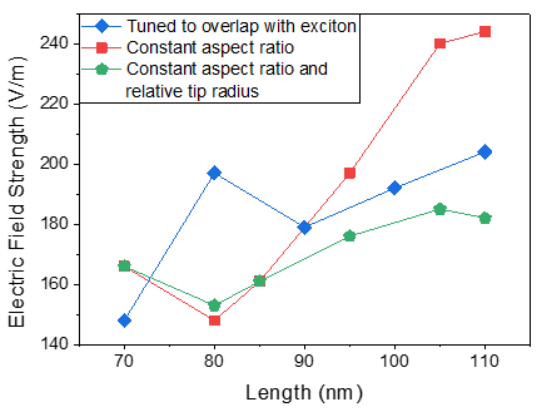

(h)

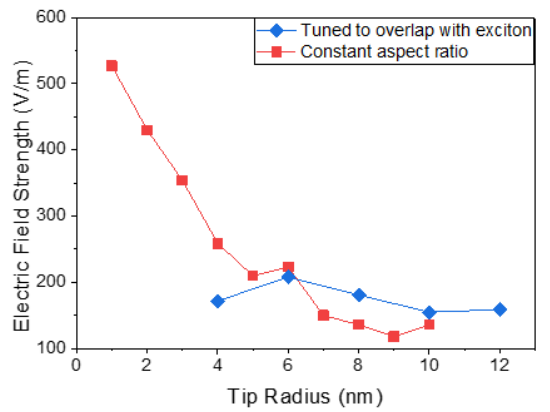

Figure 4. (a) and (b) Simulated scattering and absorption spectra of bipyramids, coupled to $\mathrm{MoS}_{2}$, of length 70-110 nm and fixed tip radius of $7 \mathrm{~nm}$, with adjusted aspect ratios to ensure zero detuning between the plasmon and exciton. (c) and (d) Typical local electric field strength maps, perpendicular to each other, (in $\mathrm{V} / \mathrm{m}$ ) of the cross section through the middle of the bipyramid and against the substrate where the bipyramid is resting respectively. The purple dashed line in figure c shows the position of the perpendicular slice shown in figure $d$ and vice versa. They are taken at the energy of highest electric field strength (energy of the exciton) White scale bars show $20 \mathrm{~nm}$.(e) Plot of the electric field strength $(\mathrm{V} / \mathrm{m})$ at the tip of different bipyramids with varying length between 70-110 $\mathrm{nm}$. The blue graph corresponds to the bipyramids presented in (a) and (b), the red curve corresponds to bipyramids with a fixed aspect ratio and fixed tip radius of $7 \mathrm{~nm}$ and the green curve corresponds to bipyramids with a fixed tip radius to width ratio and aspect ratio. (f) and (g) Simulated scattering and absorption spectra of bipyramids with varying tip radii between 4-12 $\mathrm{nm}$ and fixed length $80 \mathrm{~nm}$. The aspect ratios were adjusted to ensure zero detuning between the plasmon and exciton. (h) Plot of the electric field $(\mathrm{V} / \mathrm{m})$ at the tip of bipyramids of tip radius $2-12 \mathrm{~nm}$ corresponding to the bipyramids presented in (c) and (d) (blue) and to bipyramids of fixed length and aspect ratio (red).

in exciton energy between samples. The A exciton energy in the $\mathrm{MoS}_{2}$ was taken as the energy position of the strongest peak in the measured PL spectrum (figure 1c). $\Delta$ Energy is plotted against the energy detuning of the plasmon from the A exciton in the $\mathrm{MoS}_{2}$. Clear anti-crossing behaviour is seen in each plot, the upper and lower polaritons having higher and lower energy than the original plasmon and exciton.

A coupled oscillator model was used to fit the data obtained from the dark field scattering measurements, using equation 1 . The method of using the coupled oscillator model to extract coupling strength and Rabi splitting values from experimentally measured data is commonly used for open cavity systems. ${ }^{14,18,43,65-67}$ The energies of the upper and lower polaritons were taken as the highest points in intensity in the centre of the polariton peaks. According to Savona et al., the polaritons measured will have peaks at different values depending on the method used. The simulation data showed that the difference in the splitting between the scattering and absorption spectra was $7 \pm 4 \%$ of the total splitting calculated from the scattering spectrum. The splitting observed in the scattering spectra is always slightly higher than that from the absorption spectra. This is a small difference and shows that the coupled oscillator model can be used to estimate $g$ with a margin of error of $\sim 10 \%$.

The dissipation rates of the plasmons were estimated by averaging the full-width-half-maxima (FWHM) of the single particle scattering measurements on an $\mathrm{SiO}_{2} / \mathrm{Si}$ substrate (figure 1d). This allows for the dissipation rate of the plasmon uncoupled to $\mathrm{MoS}_{2}$ to be obtained. The $\gamma_{p l}$ was constant for all particle sizes studied. This was confirmed with FDTD simulations. The average linewidth at FWHM for all particles measured on $\mathrm{SiO}_{2} / \mathrm{Si}$ substrate was found to be $\sim 105 \mathrm{meV}$. The dissipation rate of the exciton was taken by averaging the FWHM of the PL measurements of the $\mathrm{MoS}_{2}$ flakes (figure 1e). It was found to be $50 \mathrm{meV}$. The uncoupled plasmon energy was estimated from the spectral data using ${ }^{14}$ :

$$
\omega_{p l}=\omega_{u p}+\omega_{l p}-\omega_{e x}
$$

The coupling constant, $g$, was estimated by fitting with equation 1 . This was done by finding a $g$ such that the average difference in predicted and measured polariton energies was less than $2 \mathrm{meV}$ within the sample of bipyramids. (The predicted value of polariton energy for a given $g$ was found using equation 1.) 
The dashed curved lines on the plots show the fitted upper (blue) and lower (red) polaritons, respectively, for the estimated $g$. The dashed horizontal and diagonal lines indicate the positions of the exciton and plasmon, respectively.

Figure $3 \mathrm{e}$ shows the results for bipyramids within each size category plotted together. Each size of bipyramids is given a different colour, indicating the clear trend of larger bipyramids having a stronger coupling constant. Figure $3 \mathrm{f}$ shows the trend of the coupling constant, g, increasing with bipyramid size. The coupling constant for each plot was found from the best fit with the experimental data. The Rabi splitting separation at zero detuning, $\Omega$, was also estimated from the coupling constant, using equation: ${ }^{13,47}$

$$
\Omega=\sqrt{4 g^{2}-\frac{(\Delta \gamma)^{2}}{4}}
$$

Evidence for increased splitting for larger bipyramids can also be seen in figure 2; the distance between the two polaritons is visibly greater for the larger bipyramids. Figure $3 \mathrm{e}$ shows that the Rabi splitting increases from $\sim 55 \mathrm{meV}$ for $70 \mathrm{~nm}$ long bipyramids to $\sim 80 \mathrm{meV}$ for $100 \mathrm{~nm}$ bipyramids. These values agree with those from the experimental scattering spectra (see figure S3).

Several reports indicate various criteria to achieve strong coupling including $\mathbf{g}>\gamma_{p l}, \gamma_{e x},{ }^{17,48,50} \mathbf{g}>\sqrt{\gamma_{p l} \gamma_{e x}},{ }^{17}$, and $\boldsymbol{\Omega}>$ $\frac{\gamma_{p l}+\gamma_{e x}}{2} .{ }^{14,48,49}$ In our work, $\Omega$ ranges between 50 and $80 \mathrm{meV}$, and $\gamma_{p l}$ and $\gamma_{e x}$ are $105 \mathrm{meV}$ and $50 \mathrm{meV}$, respectively. Only the bipyramids $>90 \mathrm{~nm}$ in length meet the criteria for $\Omega$, and the system does not meet the requirements for the other criteria mentioned due to the broad linewidth of the plasmon resonance. However, other evidence suggests strong coupling is occurring for all bipyramid sizes. The FWHM across the two visible peaks was measured from the scattering spectra of bipyramids of all sizes on a monolayer of $\mathrm{MoS}_{2}$ with $\sim$ zero detuning between the plasmon and exciton. The average of these was found to be $\sim 150 \mathrm{meV}$ which is significantly larger than that found for bipyramids on the $\mathrm{SiO}_{2} / \mathrm{Si}$ substrate $(\sim 105 \mathrm{mev})$. This indicates that more than an interference effect is taking place, the two separate peaks being spaced further apart. This broadening effect is visible in figures $1 \mathrm{e}$ and $\mathrm{f}$. The absorption spectra in figures 2 and 4 also support that Rabi splitting is occurring for these sizes of bipyramids. It is also clear, however, that the 70 $\mathrm{nm}$ bipyramids are on the edge of to the weak coupling regime. $\Omega \sim 50 \mathrm{meV}$ (near $\gamma_{e x}$ ), and the absorption spectra show the two peaks nearing each other in figure 4 for that size category.

The result of the coupling strength increasing with increased metal volume is in contrast with previous findings for different shaped nanoresonators. ${ }^{16,17}$ As the length of a bipyramid is increased, the volume generally also increases. In the case of the bipyramids in this study, the aspect ratio must be reduced for bipyramids of longer lengths to keep the plasmon resonance at the same energy, which further increases the volume. Therefore, the metal volume of a bipyramid with a given plasmon energy increases with length. Further simulations of the electric field elucidate the observed size dependence of the Rabi splitting. Figure $4 \mathrm{a}$ and $\mathrm{b}$ show the dependence of the coupling strength on the overall volume of the bipyramid. The length was varied within the range of the experimental data, from $70 \mathrm{~nm}$ to $110 \mathrm{~nm}$. The aspect ratio was varied to keep the zero-detuning condition between the plasmon and exciton. The overall volume was varied between $25,000 \mathrm{~nm}^{3}$ and $110,000 \mathrm{~nm}^{3}$. The tip radius was kept constant at $7 \mathrm{~nm}$ for each bipyramid size. Figure $4 \mathrm{a}$ and $b$ show the simulated scattering and absorption. The coupling strength is seen to increase by $\sim 20 \mathrm{meV}$ across the volume range, in agreement with the experimental data in figure 3.

A typical distribution of the electric field strength $(\mathrm{V} / \mathrm{m})$ in the cross section through the centre of the bipyramid is shown in figure $4 \mathrm{c}$. The electric field strength of the system was calculated using FDTD simulations. Figure 4d shows a map (perpendicular to the that in Figure 4c) of electric field strength $(\mathrm{V} / \mathrm{m})$ within the $\mathrm{MoS}_{2}$ layer where the plasmon is interacting with the exciton. Both maps are shown where the field strength is highest, at the energy of the A exciton. These electric field maps are for the $85 \mathrm{~nm}$ bipyramid in figure 2 (2.3 aspect ratio and $7 \mathrm{~nm}$ tip radius). It is clear that the electric field is enhanced and localised at the tips interacting with the $\mathrm{MoS}_{2}$. Figure 4e (blue) shows the corresponding electric field strength at the tips of the bipyramids, with spectra presented in figures $4 \mathrm{a}$ and $\mathrm{b}$. The steady upward trend mirrors the increased Rabi splitting for the larger bipyramids. There is an unexpected rise in the electric field for the bipyramid with $80 \mathrm{~nm}$ length. This can be attributed to the change in aspect ratio necessary to keep all the bipyramid plasmons energetically coincident with the exciton in $\mathrm{MoS}_{2}$. The aspect ratio must be reduced for the longer bipyramids, causing a blunting effect on the tips and lower electric field density.

The simulated electric fields at the tips of the bipyramids from figure 2 were also investigated (see red line in figure $4 \mathrm{e}$ ). The lengths of these bipyramids also varied from 70-110 nm, and had a $7 \mathrm{~nm}$ tip radius, but this time had a constant aspect ratio of 2.3. Here, there is a steady increase in the electric field. (The highest electric field strength for the $70 \mathrm{~nm}$ bipyramid is at 636 $\mathrm{nm}$, not overlapping with the exciton energy as is true of the other bipyramids investigated. This explains why the E field strength is slightly higher than expected for this simulation.) A possible reason for the increase of electric field strength with bipyramid length is that the larger bipyramids are closer to the ideal bipyramid shape. This is because, experimentally, there is a limit to how sharp the bipyramid tips can be. Thermodynamically, it would not be possible for them to be less than a few nanometers. ${ }^{68}$ Therefore, for the smaller bipyramids, the tip would be a more significant proportion of the overall width of the bipyramid, making it more rod-like. A third simulation was carried out to investigate this (see figure $4 \mathrm{e}$ green line). In the simulation, the ratio of the tip radius to overall bipyramid width was kept constant. The aspect ratio was again fixed at 2.3 . The proportions of the $85 \mathrm{~nm}$ bipyramid from figure 2 were chosen. A steady increase in electric field is observed, but less than what was found by keeping the tip radius fixed. The radius is eventually large enough for the $110 \mathrm{~nm}$ case for the field strength to decrease. The smallest bipyramid exhibits a larger electric field strength than might be expected. This is due to the very sharp tips required to keep the same proportions as the other bipyramids, significantly reducing the resonance mode volume. This result suggests that the ratio of overall width to tip is not the only factor influencing the increased field in the tips of the larger bipyramids. The plasmon within the larger bipyramids is stronger due to the extra electrons present in the larger metal volume. The field enhancement, however, is still concentrated at the same equally sharp tips, resulting in a stronger coupling for the larger bipyramids. This would also explain why the opposite effect was seen in the study by Antosiewicz et al., ${ }^{17}$ in which case the simulations considered spheres, without sharp tips to further concentrate the electric field. 
For larger bipyramids, the mode volume is seen to increase slightly, due to a stronger plasmon resonance. This trend is visible in figure S4. The mode volume is determined using equation 5. The field is calculated using FDTD simulations and integrated over the volume of the $\mathrm{MoS}_{2}$ underneath the bipyramid. However, the larger bipyramids used in this study have equally sharp tips as the small ones, and the aspect ratio of the larger bipyramids is reduced in order to have the plasmon resonance at the same energy as the A exciton of $\mathrm{MoS}_{2}$. Both of these factors allow for the electric field to be aligned more directly into the surface (and therefore into the $\mathrm{MoS}_{2}$ ) when the bipyramid is larger. This effect is further elucidated in the schematic in figure S5. This direct alignment focuses the E field into the $\mathrm{MoS}_{2}$, concentrating it into a smaller mode volume. A large aspect ratio or large tip radius results in the electric field overlapping the $\mathrm{MoS}_{2}$ at a more oblique angle. This causes the electric field to overlap less strongly and with a larger area of $\mathrm{MoS}_{2}$, increasing the mode volume. This effect can be seen in figure $\mathrm{S} 4$, as the mode volume is shown to decrease when the bipyramid reaches $\sim 90 \mathrm{~nm}$ in length when the bipyramids are tuned to overlap the A exciton. When a constant aspect ratio is kept, a similar trend is seen, due to the tip radius being kept constant (so lower in proportion to the rest of the bipyramid). The rise in mode volume with bipyramid length is only seen consistently when the aspect ratio and tip radius are adjusted with the length to keep the proportions of the bipyramid the same. When the length is kept constant, but the tip radius is increased, a clear trend of increased mode volume is seen, even when the aspect ratio is reduced to overlap the plasmon with the A exciton, as is seen in figure $\mathrm{S} 4(\mathrm{~b})$.

Figure S6 elucidates how the mode volume changes outside the $\mathrm{MoS}_{2}$ layer. The mode volume at the tip increases for longer bipyramids, but only very slightly. The difference is more significant when the tip radius is increased, with the mode volume larger for bipyramids with larger tip radii.

The importance of the sharpness of the tips was also investigated, as shown in figure $4 \mathrm{f}$ and $\mathrm{g}$. The length of each bipyramid was kept constant, at $80 \mathrm{~nm}$. As in the previous example, the aspect ratio was adjusted slightly for each simulation to maintain zero-detuning between the plasmon and exciton. The aspect ratio was increased for the bipyramids with larger tips to prevent the plasmon from blue shifting. A clear trend is visible as the tip radius was increased from $4 \mathrm{~nm}$ to $12 \mathrm{~nm}$ as the splitting decreases by $35 \mathrm{meV}$. There are two reasons for this difference. As the tip gets less sharp, the nanoparticle gets more rod-like, causing the resonance to spectrally broaden, increasing $\gamma_{\mathrm{pl}}$, the plasmon line width. According to equation 2, when $\gamma_{\mathrm{pl}}$ gets too big, the system transitions to the weak coupling regime. This is visible in the absorption spectra (figure $4 \mathrm{~g}$ ) as the tip is increased past $10 \mathrm{~nm}$, the two polaritons are no longer distinguishable from each other. Another factor contributing to this effect is the increased mode volume of the resonator with increased tip radius. This also reduces the coupling strength, according to equations 3 and 4. A decrease in electric field is observed for larger tips, as expected (see figure $4 \mathrm{~h}$, blue line). It is slightly lower than expected for the $4 \mathrm{~nm}$ tip radius, attributed to the low aspect ratio required to blue-shift the plasmon to the correct energy. The results emphasize the importance of sharp tips to achieve larger splitting between the polaritons.

In the case where the aspect ratio is fixed while the tip radius changes from $1 \mathrm{~nm}$ to $10 \mathrm{~nm}$ (see figure $4 \mathrm{~h}$, red line), the results are as expected. The field decreases with a larger tip radius and gets very large when the radius is reduced to unfeasibly small sizes. The field for the $6 \mathrm{~nm}$ tip radius is higher than expected. Upon further investigation, it was observed that this is the bipyramid with closest to zero detuning between its plasmon and the exciton. It therefore has the strongest interaction with the $\mathrm{MoS}_{2}$, resulting in a slightly larger electric field.

Figures $4 \mathrm{e}$ and $\mathrm{h}$ show some bipyramids with the highest electric field strength being at a different energy to that of the A exciton. These are the $70 \mathrm{~nm}$ long bipyramid in figure 4e (red line) and the bipyramids of tip radii $1,2,3,4,5$ and 10 in figure 4h (red line). The highest electric field strength for each of these bipyramids is higher than it would be if it were more strongly coupled to the $\mathrm{MoS}_{2}$ layer (but the peak of high intensity has a lower FWHM). This effect is visible in figures $4 \mathrm{e}$ and h.

The electric field strength directly under the bipyramid's tip within the CTAB-mimicking layer and $\mathrm{MoS}_{2}$ layer was also investigated (see figure S7). This was to show how the modal field's overlap with the ultrathin $\mathrm{MoS}_{2}$ layer depends on the bipyramid length and tip radius. The average electric field strength within these layers was shown to increase as the bipyramid length increased or the tip radius decreased. Further, the electric field strength was shown to penetrate further through the CTAB layer and into the substrate, hence resulting in stronger interaction with the $\mathrm{MoS}_{2}$ monolayer, for longer lengths and smaller tip radii. This gives further evidence as to how larger bipyramids with sharp tips produce stronger coupling in this system.

\section{Conclusions}

In conclusion, we have investigated the dependence of Rabi splitting on bipyramid shape and dimensions. It is shown that the coupling strength is strongly influenced by the both tip radius and the length of the bipyramid being used. The tip sharpness plays a crucial role, with a transition to the weak coupling regime if not sharp enough. To benefit from the largest field enhancements and the largest Rabi splitting, for a bipyramid with a fixed aspect ratio, very sharp tips with radii of less than $7 \mathrm{~nm}$ are required. The dependence of the field strength on the bipyramid length has also been clearly observed. The localization of the field enhancement at the tips makes larger bipyramids preferable to achieve strong coupling. Rabi splitting is observed to increase from $\sim 55 \mathrm{meV}$ with a $70 \mathrm{~nm}$ long bipyramid to $\sim 80 \mathrm{meV}$ for a $100 \mathrm{~nm}$ long bipyramid. We have shown how the coupling strength in a Rabi splitting system can be enhanced by increasing the metal volume of the nanoantennae. This has not been achieved before without also increasing the number of excitons coupled into the system. This study has shown that the shape of the nanoresonator must be carefully considered when choosing the ideal size of nanoparticle to achieve strong coupling.

\section{Methods}

Growth of $\mathrm{MoS}_{2}$ : Monolayer $\mathrm{MoS}_{2}$ flakes were grown by chemical vapour deposition (CVD) on a $300 \mathrm{~nm} \mathrm{SiO}_{2}$ layer on a $\mathrm{Si}$ substrate. A target substrate $\left(\mathrm{SiO}_{2} / \mathrm{Si}\right)$ was placed face-down on a seed substrate (liquid-phase exfoliated $\mathrm{MoO}_{3}$ nanosheets drop cast onto a $300 \mathrm{~nm}$ layer of $\mathrm{SiO}_{2}$ on $\mathrm{Si}^{69}$ ). This formed a microreactor which was placed in the centre of a quartz tube furnace. It was heated to $750{ }^{\circ} \mathrm{C}$ under 150 standard cubic centimetres per minute $(\mathrm{sccm})$ Ar flow at a pressure of $\sim 0.7$ Torr. Sulfur 
vapour was generated by heating sulfur powder to $\approx 120{ }^{\circ} \mathrm{C}$ in a separate downstream part of the furnace. The microreactor was exposed to the sulfur vapour for the growth of monolayer $\mathrm{MoS}_{2}$ flakes, with forming gas as a carrier. ${ }^{56}$

Photolithography: Photolithography was carried out so the dark field spectra of individual gold bipyramids could be correlated with their corresponding SEM images. It was done with an OAI mask aligner. The photoresist and photodeveloper used were S1813 resist and MF-319 developer respectively. The metal used was a $5 \mathrm{~nm}$ adhesion layer of Ti and $35 \mathrm{~nm}$ Au. NMP 1165 developer, acetone and isopropyl alcohol were used to etch away the metal.

Bipyramid Synthesis: Au penta-twinned seeds were synthesised. ${ }^{57}$ A $10 \mathrm{~mL}$ solution containing $0.125 \mathrm{mM} \mathrm{HAuCl}_{4}$ and $0.25 \mathrm{mM}$ sodium citrate was prepared. $0.15 \mathrm{~mL}$ of $10 \mathrm{mM}$ $\mathrm{NaBH}_{4}$ was added. This changed the solution from colourless to slightly pink. The seeds were aged for two hours before use. The solution to grow the bipyramids out of seeds was prepared ${ }^{57}$ using a $0.1 \mathrm{M}$ cetyl trimethylammonium bromide (CTAB) solution. $0.5 \mathrm{~mL}$ of a $10 \mathrm{mM} \mathrm{HAuCl}_{4}$ and $0.1 \mathrm{~mL} 10 \mathrm{mM} \mathrm{AgNO}_{3}$ were added. $0.2 \mathrm{~mL}$ of $1.0 \mathrm{M} \mathrm{HCl}$ was added to acidify the solution. $0.08 \mathrm{~mL}$ of $0.1 \mathrm{M}$ ascorbic acid was added to reduce the $\mathrm{Au}(\mathrm{III})$ to $\mathrm{Au}(\mathrm{I}) .80 \mu \mathrm{L}$ of seeds were added to the solution. The reaction was carried out in a $30{ }^{\circ} \mathrm{C}$ water bath for two hours, until the solution turned purple. The concentration of the seeds and $\mathrm{HAuCl}_{4}$ solution were varied to change the overall volume of the bipyramids. The concentrations of the ascorbic acid and $\mathrm{AgNO}_{3}$ solutions were varied to alter the aspect ratios. The bipyramids were diluted in ethanol before dropping onto the CVD-grown $\mathrm{MoS}_{2}$. All chemicals were purchased from Sigma Aldrich.

$\mathrm{MoS}_{2}$ Characterisation: The $\mathrm{MoS}_{2}$ was excited with a $532 \mathrm{~nm}$ laser. The photoluminescence spectra were recorded with an Andor 230i spectrometer. The Raman spectra were recorded with a Horiba Jobin Yvon Raman spectrometer. A 100× objective was used for both sets of measurements.

Dark-Field Measurements: The dark field measurements were taken with a dark field microscope and a $100 \times$ objective. The measurements were taken with an Andor CCD camera and an Andor 230i spectrometer.

Scanning Electron Microscopy: The SEM images were taken with a Zeiss Ultra scanning electron microscope, operating at 5 $\mathrm{kV}$. Measurements were made using the InLens detector. Adhesive electrically conductive paint, silver loaded form RS Pro was used as a conductive layer.

Numerical Simulations: The finite-difference-time-domain method with FDTD solutions, Lumerical, was used. This was used to calculate the scattering, absorption, electric field strength and modal volume of the system. The dimensions of the bipyramids were taken from the corresponding SEM images. The bipyramids were approximated as two cones connected at their base (allowing for the rounding effect seen in nature with particles that size). See figure S8. The bipyramid is placed on a substrate as in experiments $-0.5 \mathrm{~nm}$ CTABmimicking layer (with an refractive index of $n=1.45^{70}$ ), $0.75 \mathrm{~nm}$ thick of monolayer $\mathrm{MoS}_{2}, 300 \mathrm{~nm}$ layer thick $\mathrm{SiO}_{2}$ and $1 \mathrm{~mm}$ layer $\mathrm{Si}$. The thickness of the CTAB layer was chosen as it most closely resembled the experimental data. The permittivity of monolayer $\mathrm{MoS}_{2}$ and $\mathrm{Au}$ is taken from Jung et al. ${ }^{64}$ and Johnson and Christy ${ }^{71}$ respectively. The TMDC was modelled as an isotropic material. The bipyramid is meshed with $1 \mathrm{~nm}$ resolution, with $0.5 \mathrm{~nm}$ resolution at the tips and $0.375 \mathrm{~nm}$ and $0.2 \mathrm{~nm}$ resolution along the thickness of the $\mathrm{MoS}_{2}$ layer and CTAB layer respectively.

\section{ASSOCIATED CONTENT}

\section{Supporting Information}

Examples of experimental scattering of single bipyramids on the $\mathrm{SiO}_{2} / \mathrm{Si}$ substrate for varying sizes and aspect ratios, simulated dependence of the scattering and absorption spectra on the A and B exciton of $\mathrm{MoS}_{2}$, examples of experimental scattering of bipyramids of various sizes coupled to a monolayer of $\mathrm{MoS}_{2}$ at zero detuning, simulated mode volume dependence on bipyramid length and tip radius, explanation of mode volume dependence on bipyramid length and tip radius, electric field dependence on bipyramid length and tip radius, simulated average electric field strength within the $\mathrm{MoS}_{2}$ and CTABmimicking layer and as a ratio of $\mathrm{MoS}_{2}$ to the CTAB-mimicking layer for bipyramids of various lengths and tip radii, comparison of simulated scattering spectra of bipyramids modelled with two cones and bipyramids modelled with ten sides.

This material is available free of charge via the Internet at http://pubs.acs.org.

\section{AUTHOR INFORMATION}

\section{Corresponding Author}

* Email: bradlel@tcd.ie

\section{Author Contributions}

All authors have given approval to the final version of the manuscript.

\section{Funding Sources}

This work was supported by Science Foundation Ireland (SFI) under Grant Numbers 16/IA/4550, 17/RC-PhD/3484 and 15/SIRG/3329.

Notes

The authors declare no competing financial interest.

ORCID

Julia Lawless: 0000-0003-1472-2218

Calin Hrelescu: 0000-0002-2410-4512

Carolyn Elliott: 0000-0002-9197-8906

Lisanne Peters: 0000-0002-0551-6263

Niall McEvoy: 0000-0001-5950-8755

A. Louise Bradley: 0000-0002-9399-8628

\section{ACKNOWLEDGMENT}

The SEM imaging for this project (grant reference numbers/body) was carried out at the Advanced Microscopy Laboratory (AML) at the AMBER center, CRANN Institute (www.crann.tcd.ie/Facilities/Advanced-Microscopy-Laboratory.aspx), Trinity College Dublin, Ireland. AML is an SFI supported imaging and analysis center.

\section{REFERENCES}

(1) Purcell, E. M. Spontaneous Emission Probabilities at Radio Frequencies. Phys. Rev. 1946, 69, 681. https://doi.org/10.1007/978-1-4615-1963-8_40. 
(2) Cao, E.; Lin, W.; Sun, M.; Liang, W.; Song, Y. Exciton-Plasmon Coupling Interactions: From Principle to Applications. Nanophotonics 2018, 7 (1), 145-167. https://doi.org/10.1515/nanoph-2017-0059.

(3) Thomas, A.; George, J.; Shalabney, A.; Dryzhakov, M.; Varma, S J.; Moran, J.; Chervy, T.; Zhong, X.; Devaux, E.; Genet, C. et al. Ground-State Chemical Reactivity under Vibrational Coupling to the Vacuum Electromagnetic Field. Angew. Chemie 2016, 128 (38), 11634-11638. https://doi.org/10.1002/ange.201605504.

(4) Pang, Y.; Thomas, A.; Nagarajan, K.; Vergauwe, R. M. A.; Joseph K.; Patrahau, B.; Wang, K.; Genet, C.; Ebbesen, T. W. On the Role of Symmetry in Vibrational Strong Coupling: The Case of Charge-Transfer Complexation. Angew. Chemie - Int. Ed. 2020, 59 (26). https://doi.org/10.1002/anie.202002527.

(5) Thomas, A.; Jayachandran, A.; Lethuillier-Karl, L.; Vergauwe R. M. A.; Nagarajan, K.; Devaux, E.; Genet, C.; Moran, J.; Ebbesen, T. W. Ground State Chemistry under Vibrational Strong Coupling: Dependence of Thermodynamic Parameters on the Rabi Splitting Energy. Nanophotonics 2020, 9 (2), 249255. https://doi.org/10.1515/nanoph-2019-0340.

(6) Noda, S.; Fujita, M.; Asano, T. Spontaneous-Emission Control by Photonic Crystals and Nanocavities. Nat. Photonics 2007 1 (8), 449. https://doi.org/10.1038/nphoton.2007.141.

(7) Vasa, P.; Pomraenke, R.; Cirmi, G.; De Re, E.; Wang, W.; Schwieger, S.; Leipold, D.; Runge, E.; Cerullo, G.; Lienau, C. Ultrafast Manipulation of Strong Coupling in Metal-Molecular Aggregate Hybrid Nanostructures. ACS Nano 2010, 4 (12), 7559-7565. https://doi.org/10.1021/nn101973p.

(8) Imamoglu, A.; Awschalom, D. D.; Burkard, G.; DiVincenzo, D. P.; Loss, D.; Sherwin, M.; Small, A. Quantum Information Processing Using Quantum Dot Spins and Cavity Qed. Phys. $\begin{array}{lllll}\text { Rev. } & \text { Lett. } & \text { 1999, } & 83 & \text { (20), }\end{array}$ https://doi.org/10.1103/PhysRevLett.83.4204.

(9) Reithmaier, J. P.; Sẹk, G.; Löffler, A.; Hofmann, C.; Kuhn, S.; Reitzenstein, S.; Keldysh, L. V.; Kulakovskii, V. D.; Reinecke, T. L.; Forchel, A. Strong Coupling in a Single Quantum DotSemiconductor Microcavity System. Nature 2004, 432 (7014), 197-200. https://doi.org/10.1038/nature02969.

(10) Yoshle, T.; Scherer, A.; Hendrickson, J.; Khitrova, G.; Gibbs, H. M.; Rupper, G.; Ell, C.; Shchekin, O. B.; Deppe, D. G. Vacuum Rabi Splitting with a Single Quantum Dot in a Photonic Crystal Nanocavity. Nature 2004, 432 (7014), 200-203. https://doi.org/10.1038/nature03119.

(11) Milde, F.; Knorr, A.; Hughes, S. Role of Electron-Phonon Scattering on the Vacuum Rabi Splitting of a Single-Quantum Dot and a Photonic Crystal Nanocavity. Phys. Rev. B - Condens. Matter Mater. Phys. 2008, 78 (3), 035330. https://doi.org/10.1103/PhysRevB.78.035330.

(12) Peter, E.; Senellart, P.; Martrou, D.; Lemaître, A.; Hours, J.; Gérard, J. M.; Bloch, J. Exciton-Photon Strong-Coupling Regime for a Single Quantum Dot Embedded in a Microcavity. Phys. Rev. Lett. 2005, 95 (6), 067401. https://doi.org/10.1103/PhysRevLett.95.067401.

(13) Törmä, P.; Barnes, W. L. Strong Coupling between Surface Plasmon Polaritons and Emitters. Rep. Prog. Phys. 2015, 78 (1), 013901. https://doi.org/10.1088/00344885/78/1/013901.

(14) Stührenberg, M.; Munkhbat, B.; Baranov, D. G.; Cuadra, J.; Yankovich, A. B.; Antosiewicz, T. J.; Olsson, E.; Shegai, T. Strong Light-Matter Coupling between Plasmons in Individual Gold Bi-Pyramids and Excitons in Mono- and Multilayer $\mathrm{WSe}_{2}$. Nano Lett. 2018, 18 (9), 5938-5945. https://doi.org/10.1021/acs.nanolett.8b02652.

(15) Stete, F.; Koopman, W.; Bargheer, M. Signatures of Strong Coupling on Nanoparticles: Revealing Absorption Anticrossing by Tuning the Dielectric Environment. ACS Photonics 2017, 4 (7), 1669-1676. https://doi.org/10.1021/acsphotonics.7b00113.

(16) Munkhbat, B.; Baranov, D. G.; Bisht, A.; Hoque, M. A.; Karpiak B.; Dash, S. P.; Shegai, T. Electrical Control of Hybrid Monolayer Tungsten Disulfide-Plasmonic Nanoantenna Light-Matter States at Cryogenic and Room Temperatures. ACS Nano 2020, 14 (1), 1196-1206. https://doi.org/10.1021/acsnano.9b09684

Antosiewicz, T. J.; Apell, S. P.; Shegai, T. Plasmon-Exciton Interactions in a Core-Shell Geometry: From Enhanced Absorption to Strong Coupling. ACS Photonics 2014, 1 (5), 454-463. https://doi.org/10.1021/ph500032d.

(18) Zhang, L.; Gogna, R.; Burg, W.; Tutuc, E.; Deng, H. PhotonicCrystal Exciton-Polaritons in Monolayer Semiconductors. Nat. Commun. 2018, 9 (1), 1-8. https://doi.org/10.1038/s41467-018-03188-x.

(19) Balci, S. Ultrastrong Plasmon-Exciton Coupling in Metal Nanoprisms with J-Aggregates. Opt. Lett. 2013, 38 (21), 4498. https://doi.org/10.1364/ol.38.004498.

(20) Schlather, A. E.; Large, N.; Urban, A. S.; Nordlander, P.; Halas, N. J. Near-Field Mediated Plexcitonic Coupling and Giant Rabi Splitting in Individual Metallic Dimers. Nano Lett. 2013, 13 (7), 3281-3286. https://doi.org/10.1021/nl4014887.

(21) Wersall, M.; Cuadra, J.; Antosiewicz, T. J.; Balci, S.; Shegai, T. Observation of Mode Splitting in Photoluminescence of Individual Plasmonic Nanoparticles Strongly Coupled to Molecular Excitons. Nano Lett. 2017, 17 (1), 551-558. https://doi.org/10.1021/acs.nanolett.6b04659.

(22) Lepeshov, S.; Wang, M.; Krasnok, A.; Kotov, O.; Zhang, T.; Liu H.; Jiang, T.; Korgel, B.; Terrones, M.; Zheng, Y. et al. Tunable Resonance Coupling in Single Si Nanoparticle-Monolayer $\mathrm{WS}_{2}$ Structures. ACS Appl. Mater. Interfaces 2018, 10 (19), 1669016697. https://doi.org/10.1021/acsami.7b17112.

(23) Bisht, A.; Cuadra, J.; Wersäll, M.; Canales, A.; Antosiewicz, T. J.; Shegai, T. Collective Strong Light-Matter Coupling in Hierarchical Microcavity-Plasmon-Exciton Systems. Nano Lett. 2019, 19 (1), 189-196. https://doi.org/10.1021/acs.nanolett.8b03639.

(24) Liu, L.; Tobing, L. Y. M.; Yu, X.; Tong, J.; Qiang, B.; FernándezDomínguez, A. I.; Garcia-Vidal, F. J.; Zhang, D. H.; Wang, Q. J.; Luo, Y. Strong Plasmon-Exciton Interactions on Nanoantenna Array-Monolayer WS 2 Hybrid System. Adv. Opt. Mater. 2019, 1901002. https://doi.org/10.1002/adom.201901002.

(25) Han, X.; Wang, K.; Xing, X.; Wang, M.; Lu, P. Rabi Splitting in a Plasmonic Nanocavity Coupled to a $\mathrm{WS}_{2}$ Monolayer at Room Temperature. ACS Photonics 2018, 5 (10), 3970-3976. https://doi.org/10.1021/acsphotonics.8b00931.

(26) Wang, S.; Le-Van, Q.; Vaianella, F.; Maes, B.; Eizagirre Barker S.; Godiksen, R. H.; Curto, A. G.; Gomez Rivas, J. Limits to Strong Coupling of Excitons in Multilayer WS 2 with Collective Plasmonic Resonances. ACS Photonics 2019, 6 (2), 286-293. https://doi.org/10.1021/acsphotonics.8b01459.

(27) Geisler, M.; Cui, X.; Wang, J.; Rindzevicius, T.; Gammelgaard, L.; Jessen, B. S.; Gonçalves, P. A. D.; Todisco, F.; Bøggild, P.; Boisen, A. et al. Single-Crystalline Gold Nanodisks on WS 2 Mono- and Multilayers for Strong Coupling at Room Temperature. ACS $\begin{array}{llll}\text { Photonics } & \text { 2019, } & 6 & \text { (4), }\end{array}$ https://doi.org/10.1021/acsphotonics.8b01766.

(28) Kleemann, M. E.; Chikkaraddy, R.; Alexeev, E. M.; Kos, D.; Carnegie, C.; Deacon, W.; De Pury, A. C.; Große, C.; De Nijs, B.; Mertens, J. et al. Strong-Coupling of $\mathrm{WSe}_{2}$ in Ultra-Compact Plasmonic Nanocavities at Room Temperature. Nat. Commun. 2017, 8 (1), 1-7. https://doi.org/10.1038/s41467-01701398-3.

(29) Mak, K. F.; Lee, C.; Hone, J.; Shan, J.; Heinz, T. F. Atomically Thin $\mathrm{MoS}_{2}$ : A New Direct-Gap Semiconductor. Phys. Rev. Lett. 2010, 105 (13), 136805. https://doi.org/10.1103/PhysRevLett.105.136805.

(30) Kang, J.; Seo, J. W. T.; Alducin, D.; Ponce, A.; Yacaman, M. J.; Hersam, M. C. Thickness Sorting of Two-Dimensional Transition Metal Dichalcogenides via Copolymer-Assisted Density Gradient Ultracentrifugation. Nat. Commun. 2014, 5, 5478. https://doi.org/10.1038/ncomms6478.

(31) Duan, X.; Wang, C.; Pan, A.; Yu, R.; Duan, X. Two-Dimensional Transition Metal Dichalcogenides as Atomically Thin Semiconductors: Opportunities and Challenges. Chem. Soc. Rev. 2015, 44 (24), 8859-8876. https://doi.org/10.1039/c5cs00507h.

(32) Mak, K. F.; Shan, J. Photonics and Optoelectronics of 2D Semiconductor Transition Metal Dichalcogenides. Nat. $\begin{array}{llll}\text { Photonics } & \text { 2016, } & 10 & \text { (4), }\end{array}$ 
https://doi.org/10.1038/nphoton.2015.282.

Lebègue, S.; Eriksson, 0. Electronic Structure of TwoDimensional Crystals from Ab Initio Theory. Phys. Rev. B Condens. Matter Mater. Phys. 2009, 79 (11), 115409. https://doi.org/10.1103/PhysRevB.79.115409.

(34) Kuc, A.; Zibouche, N.; Heine, T. Influence of Quantum Confinement on the Electronic Structure of the Transition Metal Sulfide TS2. Phys. Rev. B - Condens. Matter Mater. Phys. 2011, $83 \quad$ (24), 245213. https://doi.org/10.1103/PhysRevB.83.245213.

(35) Cheiwchanchamnangij, T.; Lambrecht, W. R. L. Quasiparticle Band Structure Calculation of Monolayer, Bilayer, and Bulk MoS 2. Phys. Rev. B - Condens. Matter Mater. Phys. 2012, 85 (20), 205302. https://doi.org/10.1103/PhysRevB.85.205302.

(36) Zeng, H.; Liu, G. Bin; Dai, J.; Yan, Y.; Zhu, B.; He, R.; Xie, L.; Xu, S.; Chen, X.; Yao, W. et al. Optical Signature of Symmetry Variations and Spin-Valley Coupling in Atomically Thin Tungsten Dichalcogenides. Sci. Rep. 2013, 3, 1608. https://doi.org/10.1038/srep01608.

(37) Lee, B.; Liu, W.; Naylor, C. H.; Park, J.; Malek, S. C.; Berger, J. S.; Johnson, A. T. C.; Agarwal, R. Electrical Tuning of ExcitonPlasmon Polariton Coupling in Monolayer $\mathrm{MoS}_{2}$ Integrated with Plasmonic Nanoantenna Lattice. Nano Lett. 2017, 17 (7), 4541-4547. https://doi.org/10.1021/acs.nanolett.7b02245.

(38) Baranov, D. G.; Wersäll, M.; Cuadra, J.; Antosiewicz, T. J.; Shegai, T. Novel Nanostructures and Materials for Strong Light-Matter Interactions. ACS Photonics 2018, 5 (1), 24-42. https://doi.org/10.1021/acsphotonics.7b00674.

(39) Dufferwiel, S.; Schwarz, S.; Withers, F.; Trichet, A. A. P.; Li, F.; Sich, M.; Del Pozo-Zamudio, O.; Clark, C.; Nalitov, A.; Solnyshkov, D. D. et al. Exciton-Polaritons in van Der Waals Heterostructures Embedded in Tunable Microcavities. Nat. $\begin{array}{lllll}\text { Commun. 2015, } & 6 & \text { (1), } & 1-7 .\end{array}$ https://doi.org/10.1038/ncomms9579.

(40) Liu, X.; Galfsky, T.; Sun, Z.; Xia, F.; Lin, E. C.; Lee, Y. H.; KénaCohen, S.; Menon, V. M. Strong Light-Matter Coupling in TwoDimensional Atomic Crystals. Nat. Photonics 2014, 9 (1), 30. https://doi.org/10.1038/nphoton.2014.304.

(41) Liu, W.; Lee, B.; Naylor, C. H.; Ee, H. S.; Park, J.; Johnson, A. T. C.; Agarwal, R. Strong Exciton-Plasmon Coupling in $\mathrm{MoS}_{2}$ Coupled with Plasmonic Lattice. Nano Lett. 2016, 16 (2), 1262-1269. https://doi.org/10.1021/acs.nanolett.5b04588.

(42) Liu, W.; Wang, Y.; Naylor, C. H.; Lee, B.; Zheng, B.; Liu, G.; Johnson, A. T. C.; Pan, A.; Agarwal, R. Understanding the Different Exciton-Plasmon Coupling Regimes in TwoDimensional Semiconductors Coupled with Plasmonic Lattices: A Combined Experimental and Unified Equation of Motion Approach. ACS Photonics 2018, 5 (1), 192-204. https://doi.org/10.1021/acsphotonics.7b00672.

(43) Cuadra, J.; Baranov, D. G.; Wersäll, M.; Verre, R.; Antosiewicz, T. J.; Shegai, T. Observation of Tunable Charged Exciton Polaritons in Hybrid Monolayer $\mathrm{WS}_{2}$-Plasmonic Nanoantenna System. Nano Lett. 2018, 18 (3), 1777-1785. https://doi.org/10.1021/acs.nanolett.7b04965.

(44) Zheng, D.; Zhang, S.; Deng, Q.; Kang, M.; Nordlander, P.; Xu, H. Manipulating Coherent Plasmon-Exciton Interaction in a Single Silver Nanorod on Monolayer WSe 2 . Nano Lett. 2017, $17 \quad$ (6), 3809-3814 https://doi.org/10.1021/acs.nanolett.7b01176.

(45) Liu, B.; Fathi, M.; Chen, L.; Abbas, A.; Ma, Y.; Zhou, C. Chemical Vapor Deposition Growth of Monolayer WSe 2 with Tunable Device Characteristics and Growth Mechanism Study. ACS Nano 2015, 9 (6), 6119-6127. https://doi.org/10.1021/acsnano.5b01301.

(46) Shi, H.; Yan, R.; Bertolazzi, S.; Brivio, J.; Gao, B.; Kis, A.; Jena, D.; Xing, H. G.; Huang, L. Exciton Dynamics in Suspended Monolayer and Few-Layer $\mathrm{MoS}_{2}$ 2D Crystals. ACS Nano 2013, 7 (2), 1072-1080. https://doi.org/10.1021/nn303973r.

(47) Rudin, S.; Reinecke, T. L. Anharmonic Oscillator Model for Driven and Vacuum-Field Rabi Oscillations. Phys. Rev. B Condens. Matter Mater. Phys. 2001, 63 (7), 075308. https://doi.org/10.1103/PhysRevB.63.075308.

(48) Khitrova, G.; Gibbs, H. M.; Kira, M.; Koch, S. W.; Scherer, A.
Vacuum Rabi Splitting in Semiconductors. Nat. Phys. 2006, 2 (2), 81-90. https://doi.org/10.1038/nphys227.

(49) Wang, W.; Vasa, P.; Pomraenke, R.; Vogelgesang, R.; De Sio, A.; Sommer, E.; Maiuri, M.; Manzoni, C.; Cerullo, G.; Lienau, C. Interplay between Strong Coupling and Radiative Damping of Excitons and Surface Plasmon Polaritons in Hybrid Nanostructures. ACS Nano 2014, 8 (1), 1056-1064. https://doi.org/10.1021/nn405981k.

(50) Rempe, G.; Thompson, R. J.; Kimble, H. J. Cavity Quantum Electrodynamics with Strong Coupling in the Optical Domain Phys. Scr. 1994, 1994 (T51), 67. https://doi.org/10.1088/0031-8949/1994/T51/009.

(51) Savona, V.; Andreani, L. C.; Schwendimann, P.; Quattropani, A. Quantum Well Excitons in Semiconductor Microcavities: Unified Treatment of Weak and Strong Coupling Regimes. Solid State Commun. 1995, 93 (9), 733-739. https://doi.org/10.1016/0038-1098(94)00865-5.

(52) Kewes, G.; Binkowski, F.; Burger, S.; Zschiedrich, L.; Benson, O. Heuristic Modeling of Strong Coupling in Plasmonic Resonators. ACS Photonics 2018, 5 (10), 4089-4097. https://doi.org/10.1021/acsphotonics.8b00766.

(53) Fisher, T. A.; Afshar, A. M.; Whittaker, D. M.; Skolnick, M. S.; Roberts, J. S.; Hill, G.; Pate, M. A. Electric-Field and Temperature Tuning of Exciton-Photon Coupling in Quantum Microcavity Structures. Phys. Rev. B 1995, 51 (4), 2600-2603. https://doi.org/10.1103/PhysRevB.51.2600.

(54) Koenderink, A. F. On the Use of Purcell Factors for Plasmon Antennas. Opt. Lett. 2010, 35 (24), 4208-4210. https://doi.org/10.1364/ol.35.004208.

(55) Mann, J.; Sun, D.; Ma, Q.; Chen, J. R.; Preciado, E.; Ohta, T.; Diaconescu, B.; Yamaguchi, K.; Tran, T.; Wurch, M. et al. Facile Growth of Monolayer $\mathrm{MoS}_{2}$ Film Areas on $\mathrm{SiO}_{2}$. Eur. Phys. J. B 2013, 86 (5), 226. https://doi.org/10.1140/epjb/e201331011-y.

(56) O’Brien, M.; McEvoy, N.; Hallam, T.; Kim, H. Y.; Berner, N. C.; Hanlon, D.; Lee, K.; Coleman, J. N.; Duesberg, G. S. Transition Metal Dichalcogenide Growth via Close Proximity Precursor Supply. Sci. Rep. 2014, 4, 7374 https://doi.org/10.1038/srep07374.

(57) Liu, M.; Guyot-Sionnest, P. Mechanism of Silver(I)-Assisted Growth of Gold Nanorods and Bipyramids. J. Phys. Chem. B 2005, $109 \quad$ (47), 22192-22200. https://doi.org/10.1021/jp054808n.

(58) Jana, N. R.; Gearheart, L.; Murphy, C. J. Seed-Mediated Growth Approach for Shape-Controlled Synthesis of Spheroidal and Rod-like Gold Nanoparticles Using a Surfactant Template. Adv. Mater. 2001, 13 (18), 1389-1393. https://doi.org/10.1002/15214095(200109)13:18<1389::AID-ADMA1389>3.0.CO;2-F.

(59) Lee, C.; Yan, H.; Brus, L. E.; Heinz, T. F.; Hone, J.; Ryu, S. Anomalous Lattice Vibrations of Single- and Few-Layer $\mathrm{MoS}_{2}$. ACS Nano 2010, 4 (5), 2695-2700 https://doi.org/10.1021/nn1003937.

(60) Kang, Y.; Najmaei, S.; Liu, Z.; Bao, Y.; Wang, Y.; Zhu, X.; Halas, N. J.; Nordlander, P.; Ajayan, P. M.; Lou, J. et al. Plasmonic Hot Electron Induced Structural Phase Transition in a $\mathrm{MoS}_{2}$ Monolayer. Adv. Mater. 2014, 26 (37), 6467-6471. https://doi.org/10.1002/adma.201401802.

(61) Scheuschner, N.; Gillen, R.; Staiger, M.; Maultzsch, J. Interlayer Resonant Raman Modes in Few-Layer MoS 2. Phys. Rev. B Condens. Matter Mater. Phys. 2015, 91 (23), 235409. https://doi.org/10.1103/PhysRevB.91.235409.

(62) Lundt, N.; Klembt, S.; Cherotchenko, E.; Betzold, S.; Iff, O.; Nalitov, A. V.; Klaas, M.; Dietrich, C. P.; Kavokin, A. V.; Höfling, $S$. et al. Room-Temperature Tamm-Plasmon ExcitonPolaritons with a WSe 2 Monolayer. Nat. Commun. 2016, 7 (1), 1-6. https://doi.org/10.1038/ncomms13328.

(63) Faucheaux, J. A.; Fu, J.; Jain, P. K. Unified Theoretical Framework for Realizing Diverse Regimes of Strong Coupling between Plasmons and Electronic Transitions. J. Phys. Chem. $\begin{array}{lllll}C & \mathbf{2 0 1 4}, & 118 & \text { (5), 2710-2717. }\end{array}$ https://doi.org/10.1021/jp412157c.

(64) Jung, G. H.; Yoo, S. J.; Park, Q. H. Measuring the Optical Permittivity of Twodimensional Materials without a Priori 
Knowledge of Electronic Transitions. Nanophotonics 2018, 8 (2), 263-270. https://doi.org/10.1515/nanoph-2018-0120.

(65)

Wang S; Li, S.; Chervy, T: Shalabney, A; Azzini, S; Orgiu, E. Hutchison, J. A.; Genet, C.; Samorì, P.; Ebbesen, T. W. Coherent Coupling of $\mathrm{WS}_{2}$ Monolayers with Metallic Photonic Nanostructures at Room Temperature. Nano Lett. 2016, 16 (7), $4368-4374$ https://doi.org/10.1021/acs.nanolett.6b01475.

(66) Chikkaraddy, R.; De Nijs, B.; Benz, F.; Barrow, S. J.; Scherman, O. A.; Rosta, E.; Demetriadou, A.; Fox, P.; Hess, O.; Baumberg, J. J. Single-Molecule Strong Coupling at Room Temperature in Plasmonic Nanocavities. Nature 2016, 535 (7610), 127-130. https://doi.org/10.1038/nature17974.

(67) Wen, J.; Wang, H.; Wang, W.; Deng, Z.; Zhuang, C.; Zhang, Y.; Liu, F.; She, J.; Chen, J.; Chen, H. et al. Room-Temperature Strong Light-Matter Interaction with Active Control in Single Plasmonic Nanorod Coupled with Two-Dimensional Atomic Crystals. Nano Lett. 2017, 17 (8), 4689-4697. https://doi.org/10.1021/acs.nanolett.7b01344.
(68) Rahman, D. S.; Chatterjee, H.; Ghosh, S. K. Excess Surface Energy at the Tips of Gold Nanospikes: From Experiment to Modeling. J. Phys. Chem. C 2015, 119 (25), 14326-14337. https://doi.org/10.1021/acs.jpcc.5b03944.

(69) Hanlon, D.; Backes, C.; Higgins, T. M.; Hughes, M.; O'Neill, A.; King, P.; McEvoy, N.; Duesberg, G. S.; Mendoza Sanchez, B.; Pettersson, H.; et al. Production of Molybdenum Trioxide Nanosheets by Liquid Exfoliation and Their Application in High-Performance Supercapacitors. Chem. Mater. 2014, 26 (4), 1751-1763. https://doi.org/10.1021/cm500271u.

(70) Kékicheff, P.; Spalla, O. Refractive Index of Thin Aqueous Films Confined between Two Hydrophobic Surfaces. Langmuir 1994, $10 \quad$ (5), 1584-1591. https://doi.org/10.1021/la00017a043.

(71) Johnson, P. B.; Christy, R. W. Optical Constants of the Noble Metals. Phys. Rev. B 1972, 6 (12), 4370 https://doi.org/10.1103/PhysRevB.6.4370. 